\title{
7.87 eV Laser Desorption Postionization Mass Spectrometry of Adsorbed and Covalently Bound Bisphenol A Diglycidyl Methacrylate
}

\author{
Manshui Zhou, ${ }^{a}$ Chunping $\mathrm{Wu}^{\text {a }}$ Artem Akhmetov, ${ }^{a}$ \\ Praneeth D. Edirisinghe, ${ }^{a}$ James L. Drummond, ${ }^{b}$ and Luke Hanley ${ }^{a}$ \\ a Department of Chemistry, and \\ ${ }^{\mathrm{b}}$ Department of Restorative Dentistry, University of Illinois at Chicago, Chicago, Illinois, USA
}

Bisphenol A diglycidyl methacrylate (Bis-GMA) was adsorbed onto or covalently bound to a porous silicon oxide surface. Laser desorption $10.5 \mathrm{eV}$ postionization mass spectrometry (LDPI-MS) was previously demonstrated for surface analysis of adsorbed and surface bound Bis-GMA, but signal to noise levels were low and ion fragmentation was extensive. $7.87 \mathrm{eV}$ postionization using the fluorine laser was demonstrated here for Bis-GMA. However, signal levels remained low for LDPI-MS of Bis-GMA as its ionization potential (IP) was only $\sim 7.8 \mathrm{eV}$, near threshold for single photon ionization by the $7.87 \mathrm{eV}$ fluorine laser. It is known that aromatic tagging of molecular analytes can lower the overall IP of the tagged molecular complex, allowing $7.87 \mathrm{eV}$ single photon ionization. Therefore, Bis-GMA was also derivatized with several tags whose IPs were either below or above $7.87 \mathrm{eV}$ : the tag with an IP below 7.87 $\mathrm{eV}$ enhanced single photon ionization while the tags with higher IPs did not. However, signal intensities were enhanced by resonant laser desorption for two of the derivatized Bis-GMAs. Intact ions of Bis-GMA and its derivatives were generally observed by $7.87 \mathrm{eV}$ LDPI-MS, consistent with the formation of ions with relatively little internal energy upon threshold single photon ionization. (J Am Soc Mass Spectrom 2007, 18, 1097-1108) (c 2007 American Society for Mass Spectrometry

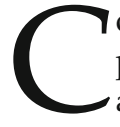
ommercial dental composites consisting of a polymerizable resin matrix, reinforcing fillers, and silane coupling agents, undergo material property changes during exposure to the oral environment [1-3]. However, examination of the organic degradation in commercial dental composites is severely limited by their complex composition of a variety of monomers and proprietary additives. Prior work by this group developed a simplified model of the dental composite that captured the essential chemical characteristics of the filler particle-silane-resin interface [4]: a polymerizable resin matrix compound bisphenol A diglycidyl methacrylate (Bis-GMA) covalently bound to a porous silicon oxide $\left(\mathrm{SiO}_{2}\right)$ surface via the silane coupling agent 3-(trimethoxysilyl) propyl methacrylate (MPS). The porous $\mathrm{SiO}_{2}$ surface was similar to that used for desorption ionization on silicon (DIOS) mass spectrometry [5].

This overlayer model system was developed to simulate the organic degradation process of commercial dental composites after aging in different media [4]. Nevertheless, surface analysis of even this relatively simple organic overlayer presented limitations for sec-

Address reprint requests to Dr. Luke Hanley, Department of Chemistry, University of Illinois at Chicago, M/C 111, 845 West Taylor St., 4500 SES, Chicago IL 60607-7061, USA.E-mail: Lhanley@uic.edu ondary ion and laser desorption mass spectrometry due to low yields and extensive fragmentation of the analyte ions. Postionization of neutral particles is a promising alternate technique to improve the ion yields, and particularly, single photon ionization (SPI) of gaseous neutrals is possible if the photon energy exceeds the neutrals' IPs. SPI has been observed for gaseous molecular species produced during laser desorption, evaporation, thermal desorption, and $\mathrm{keV}$ ion sputtering [6-21]. Laser desorption combined with various laser postionization schemes have been studied for many years and is well established $[8,9,12$, 22-24], although not as popular as other desorption/ ionization schemes. However, the recent expansion in the use of commercial atmospheric pressure photoionization sources [17] indicates the promise of vacuum ultraviolet (VUV) photoionization in vacuum for mass spectrometric applications.

A home-built laser desorption vacuum ultraviolet (VUV, $118 \mathrm{~nm}$ or $10.5 \mathrm{eV}$ ) postionization linear time-offlight mass spectrometer (LDPI-MS) was previously demonstrated for surface analysis of the aforementioned covalently bound organic overlayer [4]. However, $10.5 \mathrm{eV}$ photons are $\sim 2.7 \mathrm{eV}$ higher energy than the $\sim 7.8 \mathrm{eV}$ IP of Bis-GMA-methacryloyl overlayers (see below). This $\sim 2.7 \mathrm{eV}$ excess energy contributed to the extensive fragmentation observed during $10.5 \mathrm{eV}$ 
SPI [21]. Furthermore, the $118 \mathrm{~nm}$ pulsed radiation was generated by frequency tripling of the third harmonic of a Nd:YAG laser in pure Xe gas $[6,7]$, but the VUV intensity was only in the $\mathrm{nW}$ range and generated spectra with relatively low signal to noise.

The commercially available molecular fluorine laser is one of the most convenient laboratory sources of VUV radiation, generating $157 \mathrm{~nm}$ or $7.87 \mathrm{eV}$ photons. The $\mathrm{mW}$ power range of the fluorine laser makes it perhaps the only laboratory VUV source of sufficient intensity to saturate single photon ionization (SPI) in molecular species [25]. Its $7.87 \mathrm{eV}$ radiation can be used for threshold ionization of various classes of species including aromatic sulfides, aromatic amines, polyaromatic hydrocarbons, some pharmaceutical compounds, and tryptophan-containing peptides [20, 21, 25, 26]. Threshold ionization has the added advantage of imparting relatively little excess energy into the cation, limiting the extent of fragmentation.

One major limitation of the use of the fluorine laser for SPI is that its $7.87 \mathrm{eV}$ photon energy lies below the IPs of many interesting molecular analytes [25]. Previous work demonstrated that aromatic tagging can be used to facilitate resonant multiphoton ionization of analytes whose underivatized counterparts cannot be similarly detected [27-30]. Several of the authors previously used a similar strategy to show that aromatic tagging of molecular analytes lowers the overall IP of the tagged molecular complex, allowing $7.87 \mathrm{eV}$ SPI [26, 31-33]. Whether by single or multiphoton excitation, apparently aromatic tags can serve as chromophores to allow photoionization [10]. Furthermore, aromatic chemical tags such as anthracene that lower the IP also delocalize charge across the radical cation and permit—in at least some cases [26, 32] — the formation of intact parent ions.

The work reported herein explored the use of the fluorine laser for SPI of laser desorbed neutrals from Bis-GMA methacryloyl overlayers. The higher laser intensity of $7.87 \mathrm{eV}$ LDPI provided better signal to noise than previously observed with $10.5 \mathrm{eV}$ LDPI [4]. The $7.87 \mathrm{eV}$ LDPI-MS of Bis-GMA with derivatizing tag displaying an IP below $7.87 \mathrm{eV}$ was compared with Bis-GMA derivatized with two tags displaying IPs above $7.87 \mathrm{eV}$. The high IP tags were used to serve as controls for the hypothesis that only IP lowering tags enhance SPI. Both native and tagged Bis-GMA-methacryloyl overlayers were then prepared and analyzed using $7.87 \mathrm{eV}$ LDPIMS. These results were compared with previous work using $10.5 \mathrm{eV}$ LDPI-MS to analyze the native Bis-GMAmethacryloyl overlayer [4]. This work provides further verification of the hypothesis that tagging of analytes with low IP compounds allows their detection by 7.87 eV SPI [26, 31, 32].

These experiments were not designed to distinguish fragmentation of neutrals during laser desorption from fragmentation of parent ions during SPI. Most of the ion formation mechanisms described here assumed that entirely or mostly intact species desorbed from the surfaces, which then underwent SPI. This assumption was based upon the fact that many of the fragments of the Bis-GMA compounds displayed IPs above the photon energy, preventing their ion formation via SPI. Nevertheless, the signal observed in LDPI-MS is the net effect of both laser desorption and SPI. It was clear that some variation in laser desorption yields affected the results presented, as signal intensities were enhanced by resonant laser desorption for two of the derivatized Bis-GMAs.

Finally, X-ray photoelectron spectroscopy (XPS) was used to confirm the binding of the overlayers [4], since XPS is sensitive to elemental and functional group composition and, additionally, is more readily quantifiable for surface analysis than are mass spectrometric methods.

\section{Experimental}

\section{Materials and Sample Preparation}

4-(Phenylazo) benzoyl chloride, 4-(isocyanato) benzoyl chloride, 4-(dimethylamino) benzoyl chloride, bisphenol A diglycidyl methacrylate (Bis-GMA), 3-(trimethoxysilyl) propyl methacrylate (MPS), triethanolamine, 1-vinyl-2pyrrolidinone (VP), camphorquinone (CQ), coronene, hydrofluoric acid (HF, 48\% in water), silica gel (Davisil, grade 643, 200-524 mesh), pyridine, acetonitrile, ethyl acetate, and hexane were used as purchased (Aldrich Chemical Co., Milwaukee, WI).

Preparation of the tagged Bis-GMA-methacryloyl overlayers followed the same procedure as that of Bis-GMA-methycryloyl overlayers described previously [4]. The only difference here was that some of the overlayers were prepared using covalently tagged BisGMA, as shown in Scheme 1. The tagged Bis-GMA was synthesized through nucleophilic reaction, then separated and purified by column chromatography. The strategy to prepare the 4-(dimethylamino) benzoyl-BisGMA (referred to below as Benz-Bis-GMA) was described previously in detail [34], Ånd Ås Ånly $\AA_{\text {summa- }}$ rized here. Bis-GMA dissolved in acetonitrile and pyridine was neutralized by $\mathrm{HCl}$, then reacted with 4-(dimethylamino) benzoyl chloride under argon, stirred at room temperature overnight, and dried in a rotavapor. Benz-Bis-GMA, (Benz) ${ }_{2}$-Bis-GMA and leftover Bis-GMA were separated and purified by column chromatography with elution of ethyl acetate and hexane (2:3 ratio) on silica gel (grade 643, diameter $150 \AA$, 200-425 mesh; Aldrich Chemical Co.). Thin-layer chromatography (TLC) was used to check the separation of Benz-Bis-GMA and (Benz) ${ }_{2}$-Bis-GMA, and their structures were confirmed by proton nuclear magnetic resonance and electrospray ion trap mass spectrometry. The other tags were 4-(phenylazo) benzoyl (referred to below as Azo) and 4-(isocyanato) benzoyl (referred to below as Cyanato); their tagged-Bis-GMAs were prepared in a similar fashion. A solution of $2.0 \mathrm{mg} / \mathrm{mL}$ 

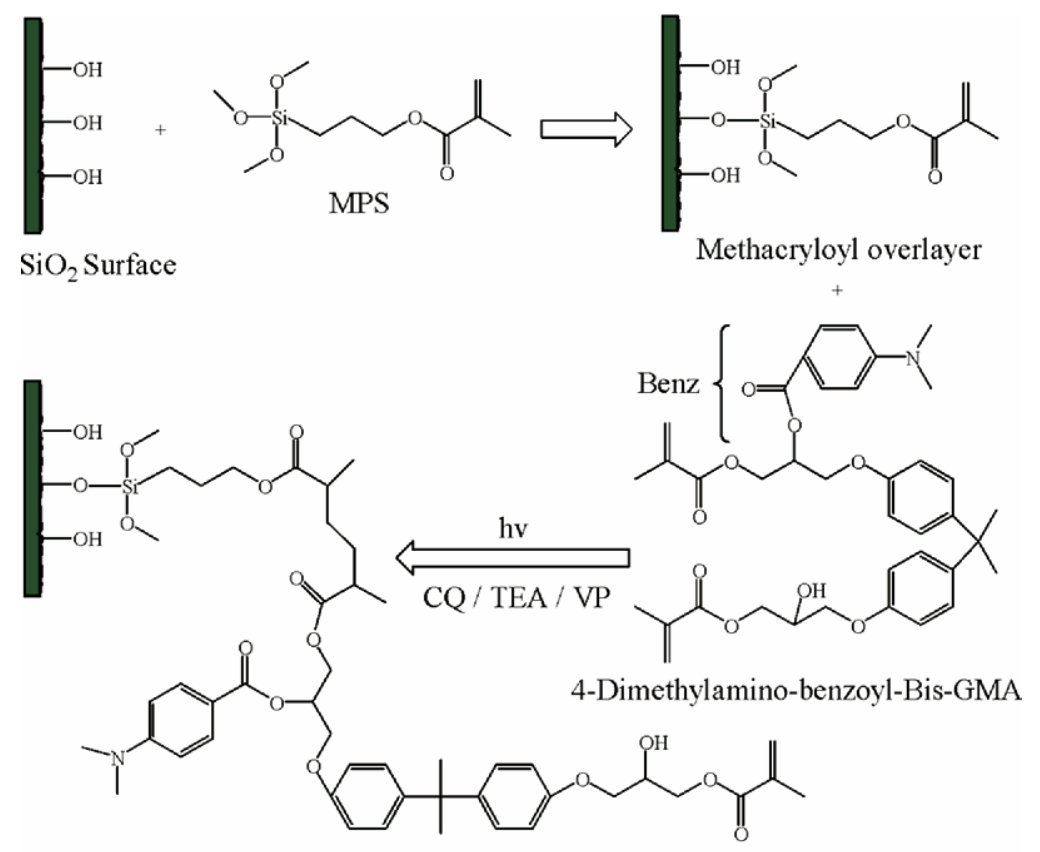

4-Dimethylamino-benzoyl-Bis-GMA-methacryloyl overlayer

Scheme 1. Schematic of the steps in preparation of the chemically derivatized overlayer model system of the dental composite; 4-dimethylamino-benzoyl-bisphenol A diglycidyl methacrylate (Bis-GMA) was bound to porous silicon oxide $\left(\mathrm{SiO}_{2}\right)$ via the silane coupling agent 3-(trimethoxysilyl) propyl methacrylate (MPS) by photoinduced functionalization with initiators (see text) to form a 4-dimethylamino-benzoyl-Bis-GMA-methocryloyl overlayer (Benz-Bis-GMA-methacryloyl overlayer).

untagged or tagged Bis-GMA in ethanol was then prepared for subsequent use as described below.

Preparation of the porous $\mathrm{SiO}_{2}$ substrate and the Bis-GMA methacryloyl overlayer was described previously $\AA 4$ ] $]$. The $\mathrm{p}$ orous $\$ \mathrm{iO}_{2}$ substrates were prepared in the Aame Áashion $\AA$ Ånose silicon wafers were etched in a 1:1 mixture of $50 \%$ aqueous HF and ethanol under ambient diffuse light, then the resultant amorphous silicon surfaces were oxidized in concentrated $\mathrm{H}_{2} \mathrm{O}_{2}$ to produce the porous $\mathrm{SiO}_{2}$ substrate. The cleaned $\mathrm{SiO}_{2}$ surfaces were silanized in MPS/toluene solution under an inert atmosphere and then baked to form the methacryloyl-coated substrate. An ethanol solution of CQ, triethanolamine, and VP co-initiators was directly added to a native or tagged Bis-GMA/ethanol solution containing the silanized substrate, which was then irradiated with visible light (Triad 2000 Visible Light Cure System; Dentsply Int., York, PA) for $20 \mathrm{~min}$.

\section{Instrumentation}

Samples were analyzed using a home built laser desorption postionization. time-of-flight mass spectrometer (LDPI-MS). Aspects of this instrument were described $\AA$ previously $\AA$ [4]. Å However, $\AA$ the $\AA$ instrument configuration was subsequently revised and described

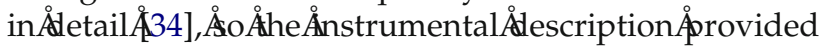
here is only a summary. The instrument is depicted schematically $\AA$ in Figure $\AA$ And $\AA$ onsisted $\AA \mathrm{f} \AA \AA$ Åad Åock, main chamber, desorption laser, postionization laser, sample holder and ion extractor, and a linear time-offlight tube with an electrostatic pulser (series EH;

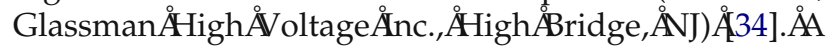
dual microchannel plate (MCP) detector ( $\mathrm{R}$. M. Jordan Co., Grass Valley, CA) with active area diameter of 18 $\mathrm{mm}$ was used to detect the ions arriving at the end of the $1.3 \mathrm{~m}$ time-of-flight tube, and the signal was processed by a digital oscilloscope (TDS5104B series; Tektronix, Portland, OR) with a bandwidth of $1 \mathrm{GHz}$, up to $16 \mathrm{MB}$ record length, and four channels. The resolution $\mathrm{m} / \Delta \mathrm{m}$, defined by the full width half maximum at the $m / z 572$ peak, was 500 and the mass accuracy was 850 ppm.

The base pressures of the vacuum system were $1 \times$ $10^{-6}$ torr in the load lock, $7 \times 10^{-9}$ torr in the main chamber, and $2 \times 10^{-9}$ torr in the time-of-flight tube. Desorption of samples was performed with a pulsed Nd:YAG laser (355 nm, Minilite II; Continuum Inc., Santa Clara, CA), which had a pulse length of $\sim 4$ ns and was focused to a power density of $\sim 3 \mathrm{MW} / \mathrm{cm}^{2}$ on the surface. The fluorine excimer laser (Optex Pro; Lambda Physik Inc., Ft. Lauderdale, FL) with wavelength of 157 nm (equivalent to a photon energy of $7.87 \mathrm{eV}$ ), pulse length of $\sim 10 \mathrm{~ns}$, and maximum energy of $1 \mathrm{~mJ} /$ pulse was used for postionization. The $157 \mathrm{~nm}$ fluorine laser beam is in the VUV range and is easily absorbed by air, so a purge tube was used to connect the fluorine laser beam exit port to the laser window on the vacuum chamber. This purge tube was pumped to vacuum by 


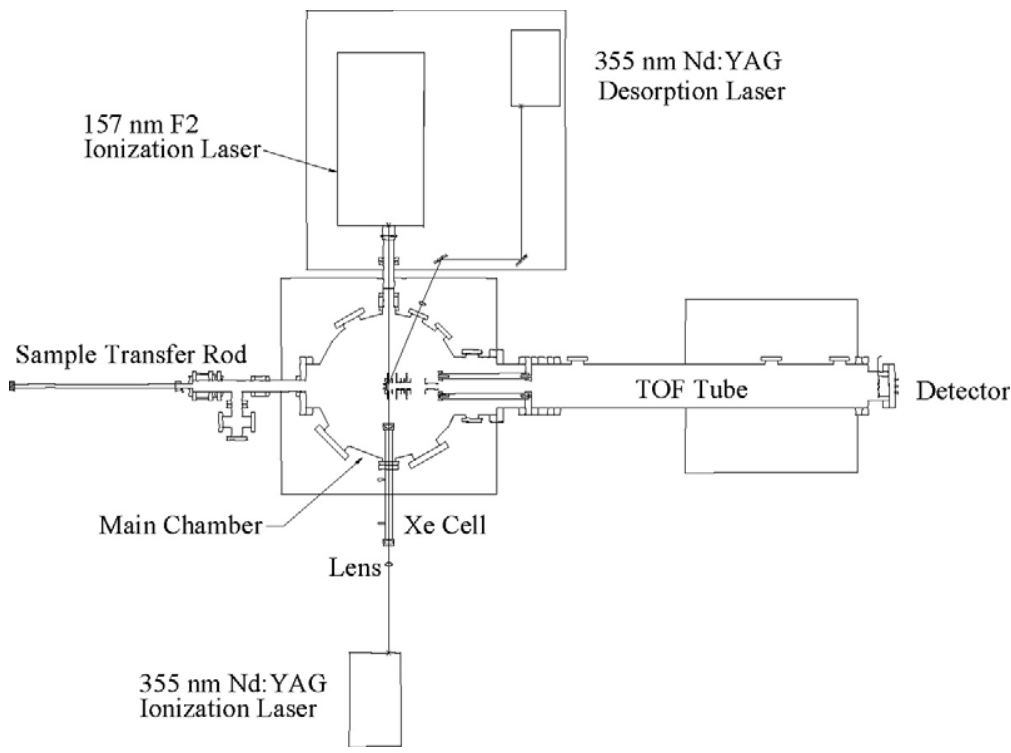

Figure 1. Diagram of the home-built $355 \mathrm{~nm}$ laser desorption (VUV, 118 and $157 \mathrm{~nm}$ ) postionization mass spectrometer (LDPI-MS) including load lock, main chamber, desorption and ionization lasers, optics, and a linear time-of-flight tube.

opening the gate valve attached to the chamber, or opening another valve attached to the load lock. A VUV grade $\mathrm{MgF}_{2}$ plano-convex lens $(35.3 \mathrm{~cm}$ focal length at $180 \mathrm{~nm}$ ) focused the $6 \times 3 \mathrm{~mm}^{2}$ dimension $157 \mathrm{~nm}$ laser beam above the sample plate, and the actual fluorine laser power was $\sim 1 \mathrm{MW} / \mathrm{cm}^{2}$ (20\% maximum power), a power range previously shown to be well within the

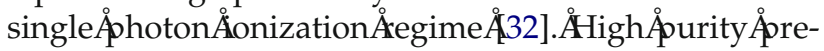
mix gas of $0.075 \%$ fluorine in helium was used for the fluorine laser discharge media.

XPS (AXIS 165; Kratos Analytical, Manchester, UK) was performed using monochromatic $1486.6 \mathrm{eV} \mathrm{Al} \mathrm{K \alpha}$ radiation $\AA$ as Ådescribed Åpreviously $\AA$ [4]. ÅTheÅsurface charge was neutralized using filament current of $1.7 \mathrm{~A}$, charge balance of $2.5 \mathrm{eV}$, and filament bias of $1.3 \mathrm{eV}$. Survey and core level scans were collected on each of at least three of every sample type. The XPS results for each of the three samples are reported as average atomic percentages \pm standard deviation, as calculated by standard methods.

\section{Data Analysis Methods}

Commercial software (ACD/MS Fragmenter; Advanced Chemistry Development Inc., Toronto, Canada) was used in electron impact and protonation modes to analyze and identify peak structures of the cations formed here by VUV postionization. This software presents fragmentation ions from a given parent ion structure as a genealogical tree through the use of established rules of ion fragmentation and was also used $\AA$ Ån.․ำ were estimated from literature values for similar com-

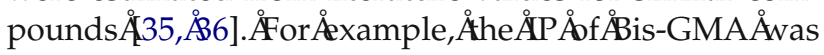
estimated as $7.8 \mathrm{eV}$ by comparison with the $7.77 \pm 0.02$
$\mathrm{eV}$ IP of the structurally similar 1-(1,1-dimethylethyl)4-methoxy benzene.

\section{Results and Discussion}

The goal of this work was to obtain high signal to noise, low fragmentation $7.87 \mathrm{eV}$ laser desorption postionization mass spectra (LDPI-MS) of the Bis-GMA methacryloyl overlayer shown in Scheme 1. However, the IP of the free Bis-GMA species was estimated to be $\sim 7.8 \mathrm{eV}$ and an isolated, laser desorbed Bis-GMA molecule in the gas phase should only be inefficiently ionized by $7.87 \mathrm{eV}$ radiation. Therefore, Bis-GMA was chemically derivatized with different chemical tags with IPs both below and above $7.87 \mathrm{eV}$ and examined by LDPI-MS. These three tags were 4-(phenylazo) benzoyl, 4-(isocyanato) benzoyl, and 4-(dimethylamino) benzyl, referred to below as Azo, Cyanato, and Benz, respectively. Next, both the underivatized and several of the tagged BisGMAs were bound via the methacryloyl silane (MPS) to porous $\mathrm{SiO}_{2}$ to form Bis-GMA methacryloyl overlayers. These overlayers were then examined by $7.87 \mathrm{eV}$ LDPI-MS and X-ray photoelectron spectroscopy (XPS). Substrate and laser desorption effects were also briefly examined for tagged Bis-GMA adsorbed on flat versus porous $\mathrm{SiO}_{2}$, as the latter was expected to enhance laser desorption since it is the standard substrate employed

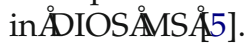

\section{Postionization of Adsorbed Species}

The results below indicated that $7.87 \mathrm{eV}$ LDPI of the native and three tagged Bis-GMA compounds proceeded via by SPI. The $\sim 7.8 \mathrm{eV}$ IP of the Bis-GMA group rendered threshold SPI via $7.87 \mathrm{eV}$ radiation 


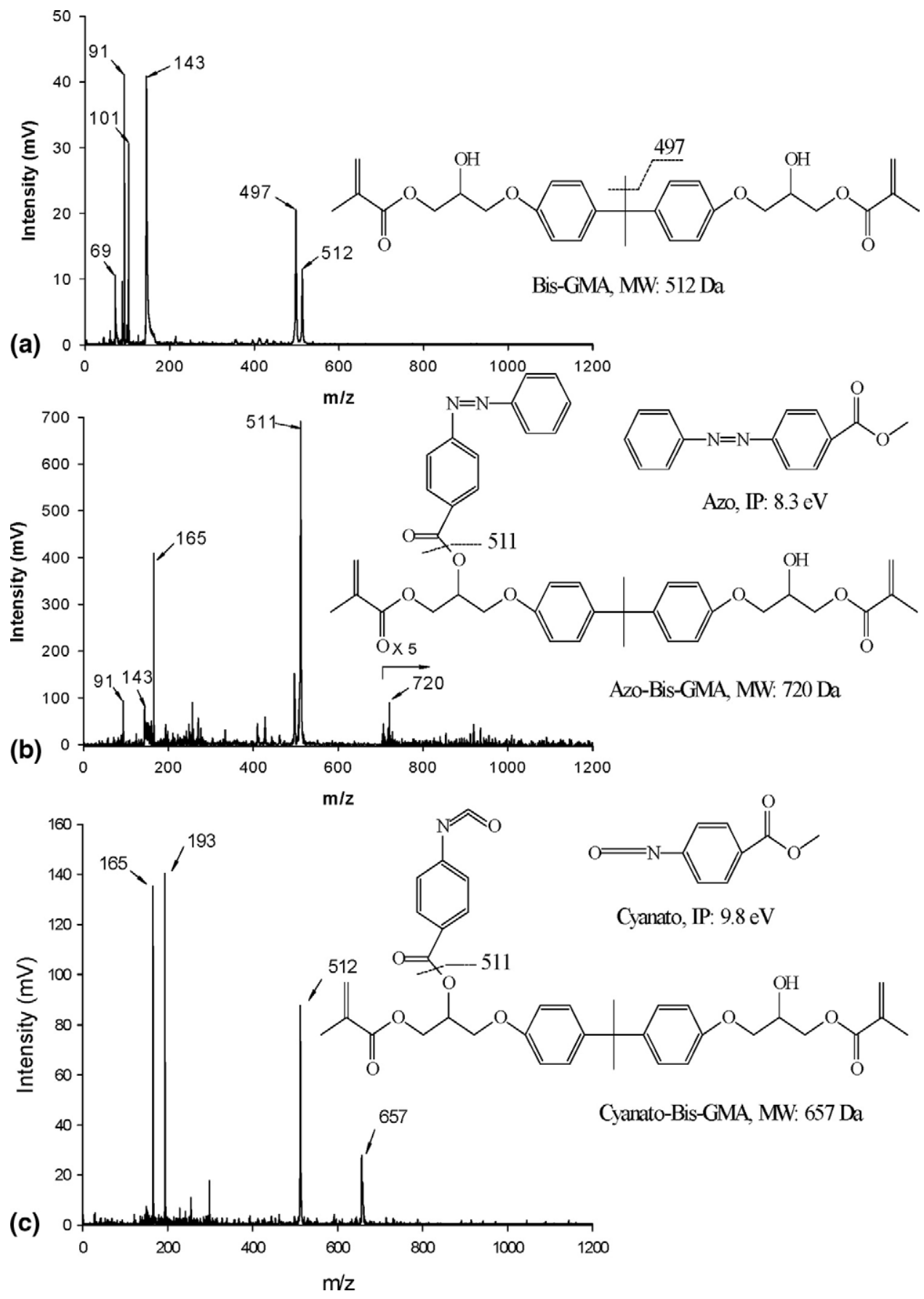

Figure 2. $355 \mathrm{~nm}$ laser desorption $7.87 \mathrm{eV}$ postionization mass spectra (LDPI-MS) of (a) Bis-GMA, (b) Azo-Bis-GMA, and (c) Cyanato-Bis-GMA adsorbed on porous $\mathrm{SiO}_{2}$. See text for experimental conditions.

possible for all the compounds and attaching the higher IP Azo or Cyanato tags did not lower the overall IP of the tagged Bis-GMA complex. However, the presence of the Benz tag with an IP of $7.4 \mathrm{eV}$ moved SPI away from threshold for Benz-Bis-GMA, dramatically increasing its ÅphotoionizationÅcross ÅsectionÅ[21] Åcompared Åwith untagged Bis-GMA. Prior experiments and ab initio calculations supported the argument that the IP of a compound is equal to or less than that of its lowest IP component $\AA$ [31, 32 32].ÅThus, Átagging Ååhigh ÅP Åanalyte with a low IP species can be used to allow SPI with relatively low photon energies. The details of these results are described further below.

The $7.87 \mathrm{eV}$ LDPI-MS of the native, Azo-, and Cyanato-tagged Bis-GMA adsorbed onto porous $\mathrm{SiO}_{2}$ areÅshownÅinÅFigure 2 2. ÅAllÅthreeÅcompoundsÅwere adsorbed in roughly equal amounts on separate porous

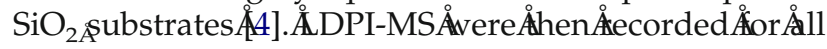
three samples under the same experimental conditions of solution concentration, desorption and ionization 
laser power, postionization delay time, time-of-flight

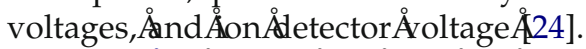

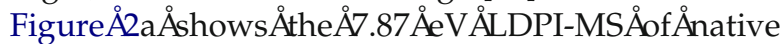
(untagged) Bis-GMA adsorbed on porous $\mathrm{SiO}_{2}$. 1-(1,1-dimethylethyl)-4-methoxy benzene, with an IP of

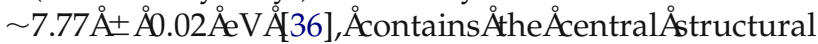
component of Bis-GMA. The intact Bis-GMA IP is therefore estimated to be $\sim 7.8 \mathrm{eV}$. Thus, Bis-GMA should undergo threshold SPI by the $7.87 \mathrm{eV}$ fluorine

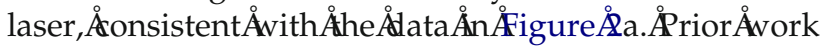
has shown that threshold SPI often forms only the

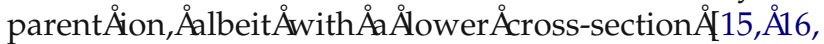

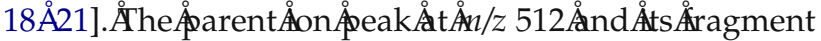
at $m / z 497$ (via loss of one methyl group) were observed using $7.87 \mathrm{eV}$ postionization, but the intensity was only $\sim 10 \mathrm{mV}$. The relatively high intensity of $\mathrm{m} / \mathrm{z} 497$ was attributable to the stability of the tertiary carbocation, which is formed in postionization. Prior analysis of Bis-GMA adsorbed on porous $\mathrm{SiO}_{2}$ using $10.5 \mathrm{eV}$ postionization showed no parent ion and was dominated instead by fragments of the parent ion after loss of $\mathrm{OH}$

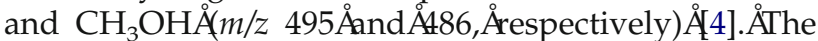
reduced parent ion fragmentation observed here at the lower photon energy is consistent with a lower internal

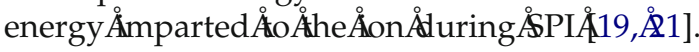

There were also several intense, but lower, mass fragment ion peaks observed in the adsorbed Bis-GMA

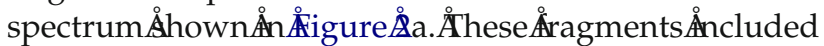
$\mathrm{m} / \mathrm{z} 69,91,101$, and 143 , most of which were attributed to the cleavage of various ether bonds in the methacrylate end group on Bis-GMA. Similar low mass ions were observed in many of the spectra presented and are discussed further below.

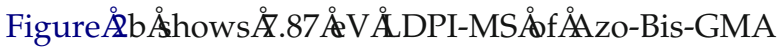
adsorbed on porous $\mathrm{SiO}_{2}$. The Azo tag structure is shown in the inset of the figure and the Azo tag IP is $\sim 8.3 \mathrm{eV}$. The parent ion peak appeared at $m / z 720$, but its intensity was relatively low compared with the dominant fragments at $m / z 511$ and lower masses. The dominant peak at $\mathrm{m} / \mathrm{z} 511$ was attributed to Bis-GMA after loss of the Azo tag, although it is unclear if this loss resulted from laser desorption or SPI. The $\mathrm{m} / \mathrm{z} 497$ peak here might have resulted from the same carbocation formation as for unlabeled Bis-GMA, but its different relative intensity indicates an alternate formation mechanism. It is speculated that cleavage of the ether bond, one atom closer to the Bis-GMA moiety, along with associated proton transfer, lead to the $\mathrm{m} / \mathrm{z} 497$ peak for Azo-Bis-GMA.

The $m / z 511$ peak from Azo-Bis-GMA showed $>60 \times$ higher signal intensity compared with the $\mathrm{m} / \mathrm{z} 512$ parent ion signal from untagged Bis-GMA. An enhancement in the SPI cross section for Azo-Bis-GMA compared with Bis-GMA was unlikely, given the $\sim 0.4 \mathrm{eV}$ higher IP for the Azo tag compared with untagged Bis-GMA. Instead, the higher signal for Azo-Bis-GMA was likely due to an enhancement in laser desorption efficiency, as discussed further in the next section. In addition to $\mathrm{m} / \mathrm{z} 91$ and 143 methacrylate fragments observed for Bis-GMA, an additional intense $\mathrm{m} / \mathrm{z} 165$ fragment appeared for Azo-Bis-GMA, which apparently resulted from a complex rearrangement.

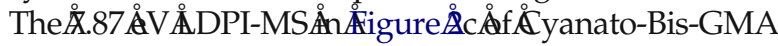
was very similar to that of Azo-Bis-GMA. The parent ion of the Cyanato-Bis-GMA at $\mathrm{m} / \mathrm{z} 657$ intensity was of the same order of magnitude as that of the Azo-tagged compound. The $\mathrm{m} / \mathrm{z} 511$ peak of Bis-GMA was also prominent in the MS of Cyanato-Bis-GMA, at much lower intensity (see next section). The high $9.8 \mathrm{eV}$ IP of the Cyanato tag did not enhance postionization of the tagged complex compared with untagged Bis-GMA, so postionization probably proceeded entirely through the Bis-GMA functionalities. The $\mathrm{m} / \mathrm{z} 165$ fragment ion apparently resulted from a complex rearrangement of the Cyanato tag.

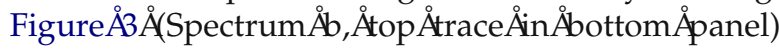
shows the 7.87 eV LDPI-MS of Benz-Bis-GMA adsorbed on porous $\mathrm{SiO}_{2}$. The IP of Benz was $7.4 \mathrm{eV}$ and this low IP led to a high photoionization cross-section, which in turn contributed to the highest signal levels seen out of all four Bis-GMA compounds. The highest mass ion peak detected was the parent ion at $\mathrm{m} / \mathrm{z} 659$ with an intensity of $540 \mathrm{mV}$. This was the highest signal observed for any of the tagged parent ions and was roughly equivalent to the signal for the $\mathrm{m} / \mathrm{z} 511$ ion of Bis-GMA observed from Azo-Bis-GMA. Laser desorption effects also contributed to the high ion signal for Benz-Bis-GMA, as discussed further in the next section.

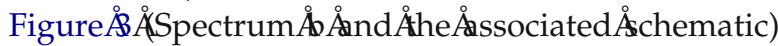
shows that the most abundant ion peak occurred at $\mathrm{m} / \mathrm{z}$ 165 and was a protonated 4-(dimethylamino) benzoyl ion or the Benz tag ion, directly cleaved from the Benz-Bis-GMA molecule. Related ions forming from the detached Benz tag included $\mathrm{m} / \mathrm{z} 135$ formed by further loss of the two methyl groups bound to the nitrogen atom and $\mathrm{m} / \mathrm{z} 149$ from protonation following loss of one oxygen atom. Cleavage at the methacrylate group on one side, the bond of ternary carbon adjacent to the closest benzene on another side formed the protonated ion peak at $m / z 193$.

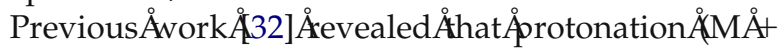
$\mathrm{H}^{+}$) following SPI was a significant channel in parent ion formation during $7.87 \mathrm{eV}$ LDPI-MS of anthracene labeled hexapeptides. High-resolution mass spectra of Benz-Bis-GMA in the region of the parent ion were collected to determine whether secondary protonation of the parent ion following its formation via SPI may have occurred here. The high-resolution LDPI-MS of

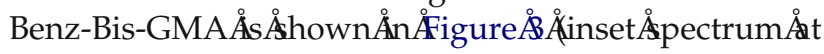
bottom right shows smoothed data): the individual mass peaks were well-separated by unit mass, allowing differentiation between SPI and protonation. The signal at $m / z 659$ was due solely to the parent ion of Benz-BisGMA, $\mathrm{M}^{+}$, containing only ${ }^{12} \mathrm{C}$ atoms. The peak $[\mathrm{M}+$ $1]^{+}$at $\mathrm{m} / \mathrm{z} 660$ was equal to the sum of the signals from the protonated parent ion containing only ${ }^{12} \mathrm{C}\left(\mathrm{M}+\mathrm{H}^{+}\right)$ and the photoionized parent ion containing ${ }^{13} \mathrm{C}$. The 


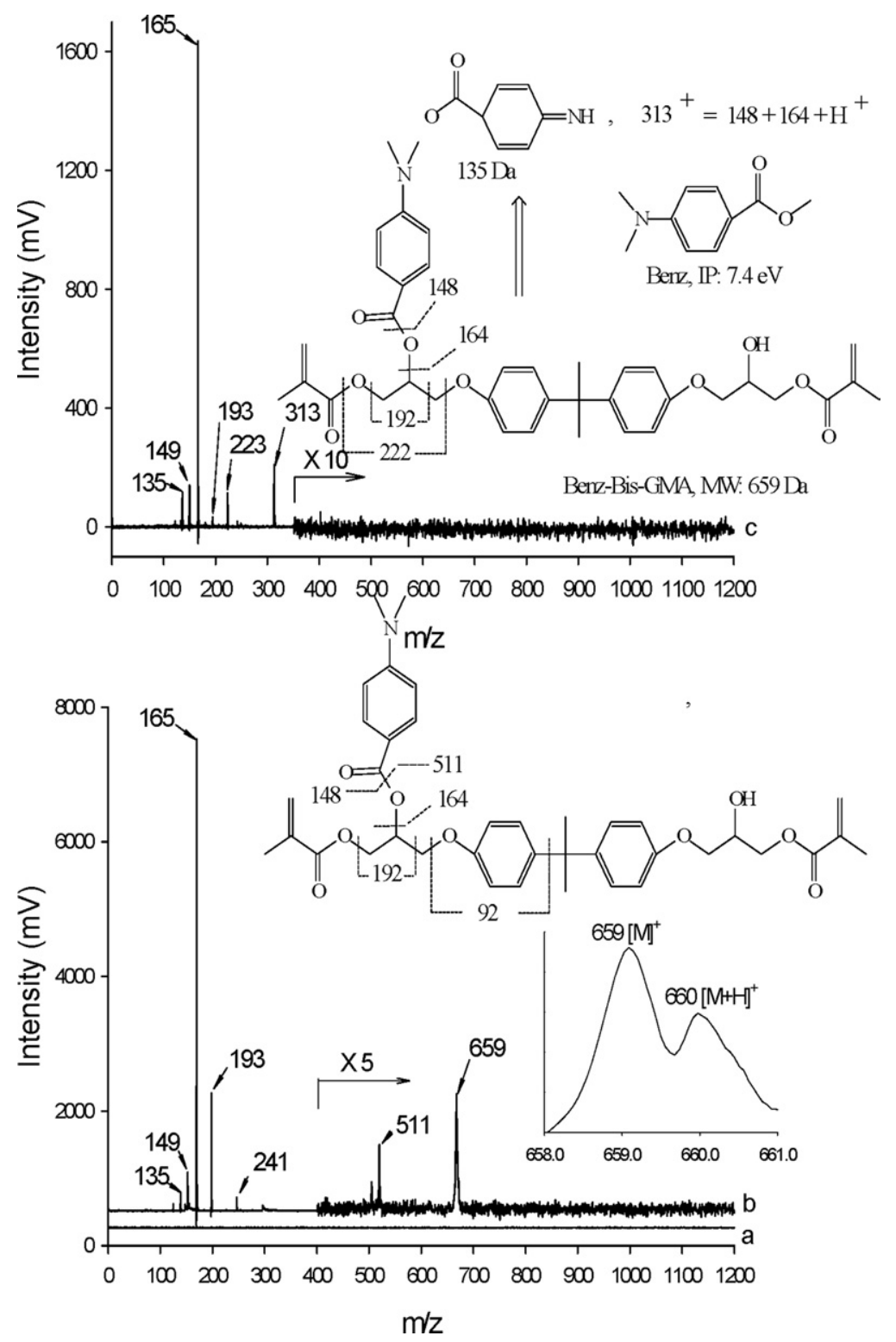

Figure 3. (a) Laser desorption MS of Benz-Bis-GMA adsorbed on porous $\mathrm{SiO}_{2}$ (without $7.87 \mathrm{eV}$ postionization, bottom trace, bottom panel). $7.87 \mathrm{eV}$ LDPI-MS of Benz-Bis-GMA adsorbed on (b) on porous $\mathrm{SiO}_{2}$ (top trace, bottom panel) and (c) flat $\mathrm{Si}$ (top panel). Inset of bottom panel is LDPI-MS on porous $\mathrm{SiO}_{2}$ except confined to $\mathrm{m} / \mathrm{z} 658$ to 661 region of parent ion and recorded at higher mass resolution. Note that letters correspond to labeled traces (a)-(c).

experimental intensity of the $\mathrm{m} / \mathrm{z} 660$ peak was $\sim 50 \%$ that of the $m / z 559$ parent ion while the isotopic contribution to this peak was $43 \%$. However, peak overlap and fitting errors (the $\mathrm{m} / \mathrm{z} 660$ peak was calculated as the average of several spectra) prevented determination of whether or not there was excess intensity in the $\mathrm{m} / \mathrm{z}$ 660 peak attributable to a proton transfer event. The most definitive statement that could be reliably made with the current data was that protonation was a minor to negligible channel for Benz-Bis-GMA. These data were collected using a newly-revised instrumental configuration with a reflectron time-of-flight MS (configuration to be discussed in a future paper).

\section{Comparison with DIOS MS: Substrate and Laser Desorption Effects}

The above discussion has focused on effects of postionization, but the laser desorption event must also be considered in the overall signal. It is well known that LDPI-MS signal is enhanced by resonant laser desorption, in which the desorption laser wavelength is one at which the analyte undergoes strong linear optical absorption $\AA$ [8]. ÅThe Ålogarithms Åof Åthe Aextinction Åcoefficients for $355 \mathrm{~nm}$ optical absorption by the desorption laser were estimated as $<1.0$ for Bis-GMA, $<1.0$ for Cyanato, 3.3 for Azo, and 3.7 for Benz by comparison 
with Similar underwent more efficient resonant laser desorption while Cyanato- and untagged Bis-GMA underwent less efficient nonresonant laser desorption. Thus, the high ion yields seen for Azo-Bis-GMA were due to enhanced laser desorption efficiency. Benz-Bis-GMA showed high ion signals due to both enhanced laser desorption efficiency and efficient SPI resulting from its low IP (see prior section). By contrast, Cyanato and untagged BisGMA underwent nonresonant laser desorption that resulted in much lower ion yields.

Azo compounds are well known to undergo photodissociation upon excitation in the ultraviolet, most typically leading to loss of $\mathrm{N}_{2(\mathrm{~g})}$ and formation of radicals, which in the case of azobenzene would be phenylÅradicals Å[37].ÅSimilarÅphotodissociationÅmay have occurred here during the laser desorption step, but no evidence for loss of intact $\mathrm{N}_{2(\mathrm{~g})}$ was observed in the Azo-Bis-GMA spectra, despite this being the dominant channel for films where the Azo group is not covalently bound $\AA 0$ 负he

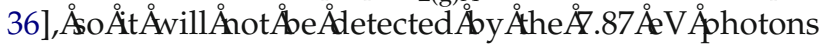
used here for postionization. Photodissociation of AzoBis-GMA may have occurred during the laser desorption step, but the results of photodissociation are not obvious in the LDPI-MS.

The porous $\mathrm{SiO}_{2}$ substrate used was similar to that employed in DIOS MS for the detection of various adsorbed Ånalytes $\AA$ [5,Å39], Åincluding Åvapor $\AA$ deposited

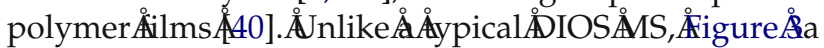
(bottom trace of bottom panel) shows almost no ion peaks appeared in the laser desorption only MS (without $7.87 \mathrm{eV}$ postionization) of Benz-Bis-GMA adsorbed on porous $\mathrm{SiO}_{2}$, where the LD density on the sample

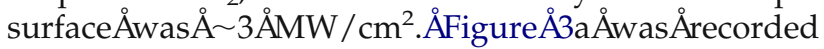
under the same laser desorption conditions used for Figure $\$ b$, was due to postionization of laser desorbed neutrals. The $1 m$ ost omplete

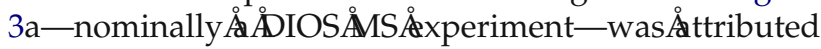
to the lower desorption laser power densities used here compared with the $>5 \mathrm{MW} / \mathrm{cm}^{2}$ values more typical in

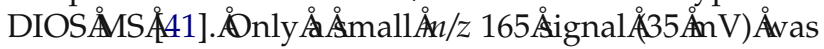
occasionally seen in the laser desorption MS, with a few

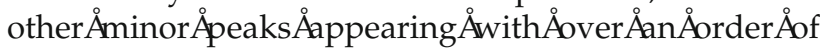
magnitude lower signal. Similarly, negligible ion signals were seen for the laser desorption MS of Bis-GMA, Azo-Bis-GMA and Cyanato-Bis-GMA (data not shown) when compared with the signals for the corresponding LDPI-MS.

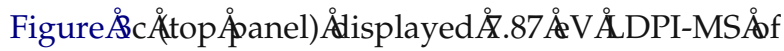
Benz-Bis-GMA on a flat $\mathrm{Si}$ wafer, with the schematic of ion formation also shown. The flat Si wafer was covered with a uniform and flat, few-nm-thick native $\mathrm{SiO}_{2}$ overlayer, unlike the highly porous $\mathrm{SiO}_{2}$ layer, which

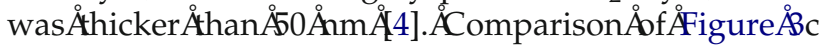
and $b$ showed that the porous $\mathrm{SiO}_{2}$ surface led to higher desorption yields of intact neutrals, with maximum ion signal from the flat surface only $20 \%$ of that from the porous surface. Similar enhancements in maximum ion signal and reduction in fragmentation were observed for Bis-GMA, Azo-Bis-GMA, and Cyanato-Bis-GMA adsorbed on porous versus flat surfaces (data not shown). However, most, if not all, of the signal enhancement may have been due to the larger surface area of the porous surface, which allowed higher surface concentrations compared with the flat surface. Enhanced desorption of intact species from the porous $\mathrm{SiO}_{2}$ surface has frequently been attributed to the well-known ability of this DIOS substrate to behave as a lightabsorbing, Alesorption-enhancing Anatrix $\AA 5, \$ 9$ ] .

There was far more fragmentation from the flat surface than there was from the porous surface, for all four compounds. As a result, $\mathrm{m} / \mathrm{z} 313$ was the highest mass ion observed for LDPI-MS of untagged BisGMAÅfrom ÅtheÅflatÅsurface $\AA$ (seeÅFigureẢ3c). ÅThe schematic Ådjacent Åo Figure fragmentation mechanisms for LDPI-MS of Benz-BisGMA from the flat surface. The ion formation for desorption from the flat surface was similar to that in

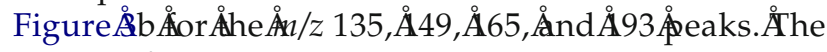
ion at $m / z 313$ was also a protonated adduct ion that formed by recombination of two fragments of the Benz tag.

\section{Analysis of Benz-Bis-GMA- and Bis-GMA- Methacryloyl Overlayers}

The results discussed above confirmed that the Benz tag improved both the desorption and ionization efficiency during $7.87 \mathrm{eV}$ LDPI-MS of Benz-Bis-GMA compared with untagged Bis-GMA. This detection advantage for Benz-Bis-GMA extended to covalently bound overlayers. Native Bis-GMA and Benz-Bis-GMA were covalently bound to the $\mathrm{SiO}_{2}$ surface via MPS through photoinduced functionalization to form native and Benz-Bis-GMA-methacrylate overlayers, respectively,

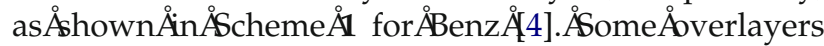
were also chemically derivatized with either two Benz tags or the Azo tag. The organic overlayers tagged with Benz showed enhanced signal and intact parent ions during $7.87 \mathrm{eV}$ LDPI-MS while those either untagged or Azo-tagged did not.

The overlayer formation was first checked by XPS,

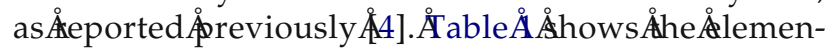
tal composition of Bis-GMA-methacryloyl overlayers that were untagged or tagged with either one or two Benz groups (the latter two denoted Benz-Bis-GMAmethacryloyl and (Benz) $)_{2}$-Bis-GMA-methacryloyl overlayers, respectively). The nitrogen content in Benz-Bis-GMA-methacryloyl overlayers was higher than that of the untagged overlayer due to the single nitrogen atom in the Benz tag. The nitrogen concentration in (Benz) $)_{2}$-Bis-GMA-methacryloyl overlayers was nearly two times as that in Benz-Bis-GMAmethacryloyl overlayers, which confirmed the covalent binding of Bis-GMA to the methacryloyl. The 
Table 1. Binding energies and elemental compositions from X-ray photoelectron spectra (XPS) of Bis-GMA-methacryloyl, Benz-Bis-GMA-methacryloyl, and (Benz) ${ }_{2}$-Bis-GMA-methacryloyl overlayers

\begin{tabular}{lcccc}
\hline \multicolumn{1}{c}{ Sample } & $\mathrm{C}_{1 \mathrm{~s}}(\%) 285 \mathrm{eV}$ & $\mathrm{O}_{1 \mathrm{~s}}(\%) 532 \mathrm{eV}$ & $\mathrm{Si}_{2 \mathrm{p}}(\%) 103 \mathrm{eV}$ & $\mathrm{N}_{1 \mathrm{~s}}(\%) 399 \mathrm{eV}$ \\
\hline \hline Bis-GMA-methacryloyl overlayers & $71.6 \pm 2.2$ & $25.3 \pm 1.9$ & $3.1 \pm 0.3$ & $1.3 \pm 0.2$ \\
Benz-Bis-GMA-methacryloyl overlayers & $60.2 \pm 2.5$ & $30.1 \pm 2.0$ & $4.8 \pm 0.5$ & $2.9 \pm 0.3$ \\
(Benz) -Bis-GMA-methacryloyl overlayers $^{\prime}$ & $62.9 \pm 2.3$ & $26.8 \pm 1.8$ & $3.9 \pm 0.4$ & $6.4 \pm 0.3$ \\
\hline
\end{tabular}

appearance of a $\mathrm{N}_{1 \mathrm{~s}}$ peak at $399 \mathrm{eV}$ for the untagged Bis-GMA-methacryloyl overlayer was due to traces of photopolymerization initiators, which were partially trapped in the overlayer during photoinduced func-

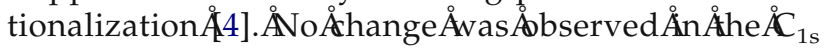
peak shape for the tagged and untagged overlayers, further indicating that the monolayers were not dramatically affected by the presence of the Benz tag (data not shown).

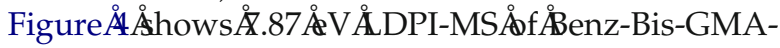
methacryloyl overlayers, along with a schematic of ion formation depicted below the spectrum. The highest mass peak from Benz-Bis-GMA-methacryloyl overlayers occurred at $m / z 773$ at the cleavage of C-Si bond by loss of one methyl from the amino group, which was consistent with the previous work using $10.5 \mathrm{eV}$

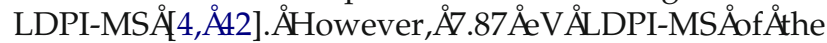
Bis-GMA-methacryloyl overlayers derivatized with Benz tags showed higher ionization efficiency, less fragmentation, and better signal to noise compared to $10.5 \mathrm{eV}$ postionization of native Bis-GMA-methacryloyl overlayers. The other major ion peaks observed were attributed to logical fragmentation pathways for the Benz-Bis-GMA-methacryloyl overlayers, as shown

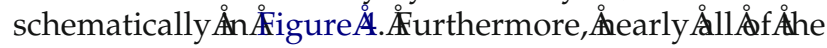
ions detected contained the substructure of the Benz tag. For example, the formation of ion peaks at $m / z 149$,

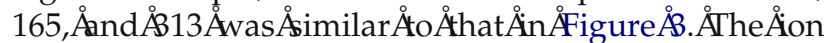
peak at $m / z 661$ resulted from the cleavage of the first ester bond adjacent to the $\mathrm{SiO}_{2}$ surface and the top
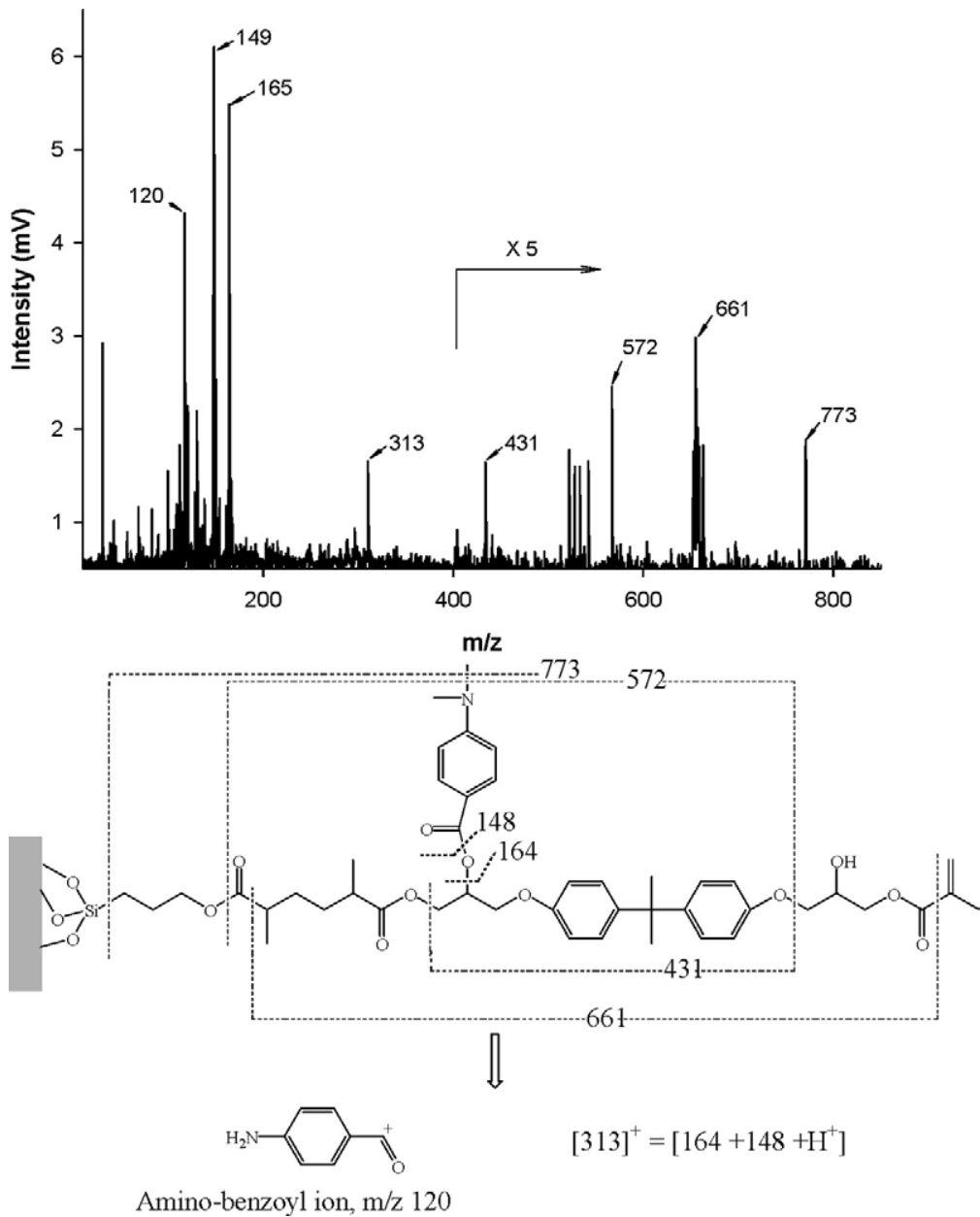

Figure 4. 7.87 eV LDPI-MS of Benz-Bis-GMA-methacryloyl overlayers, covalently bound to porous $\mathrm{SiO}_{2}$. 

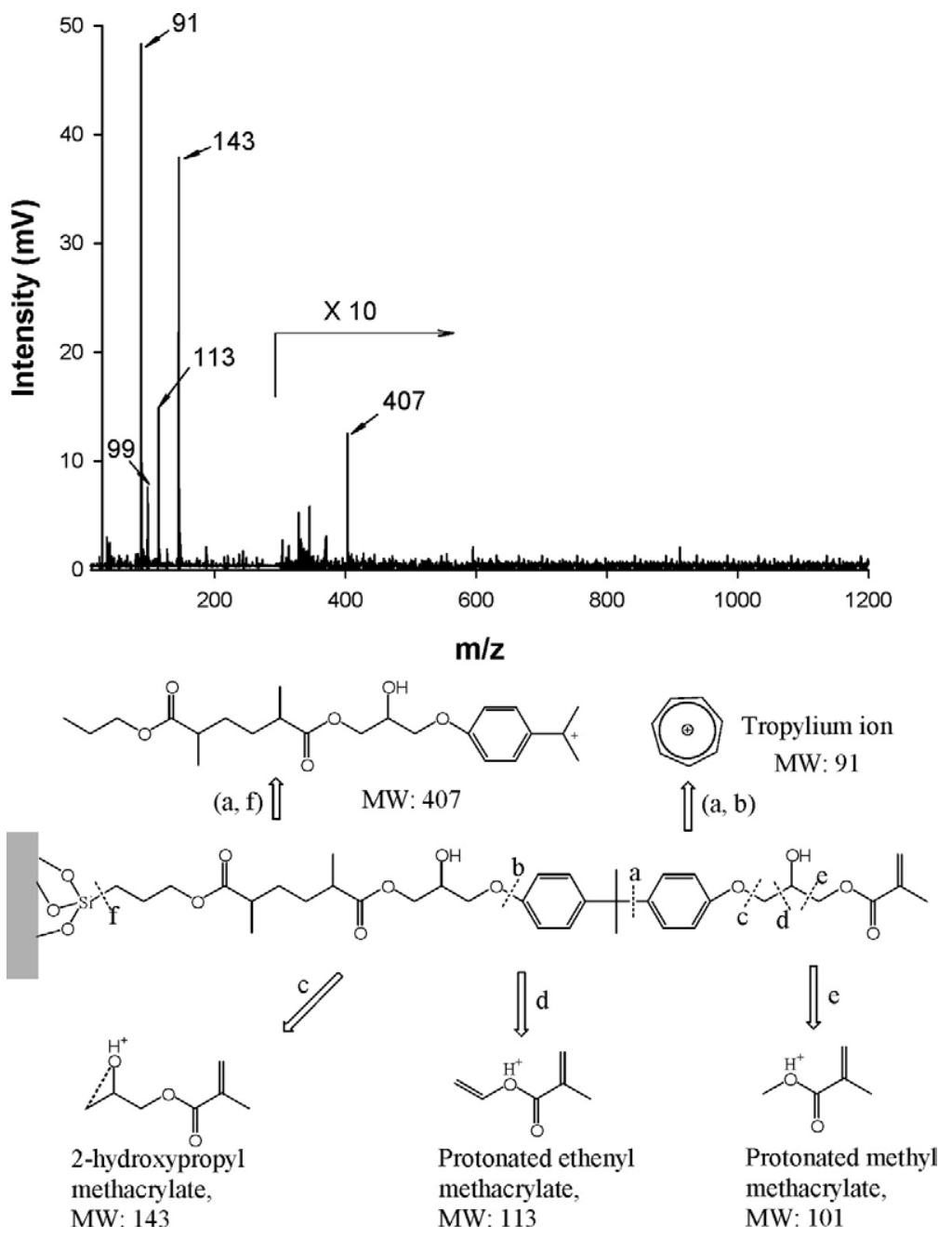

Figure 5. $7.87 \mathrm{eV}$ LDPI-MS of Bis-GMA-methacryloyl overlayers, without any attached tag, covalently bound to porous $\mathrm{SiO}_{2}$.

propene group, and the $\mathrm{m} / \mathrm{z} 572$ formed due to the cleavage of the first ester bond adjacent to the $\mathrm{SiO}_{2}$ surface and the $\mathrm{C}-\mathrm{O}$ bond adjacent to the alcohol group, the ion peak at $m / z 431$ ion formed due to the cleavage of $\mathrm{C}-\mathrm{O}$ bond of the ester group adjacent to the tag and the $\mathrm{C}-\mathrm{O}$ bond adjacent to the alcohol group.

The 7.87 eV LDPI-MS of (Benz) ${ }_{2}$-Bis-GMA-methacryloyl overlayers were also recorded (data not shown) and displayed the parent ion at $\mathrm{m} / \mathrm{z} 920$, shifted up by 147 $\mathrm{Da}$ (equal to the mass of the extra Benz tag) from the analogous ion of the singly Benz substituted parent ion

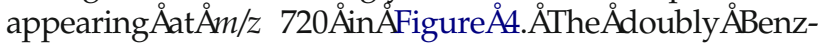
substituted overlayer also showed similar ion fragment formation as occurred for the singly substituted overlayer.

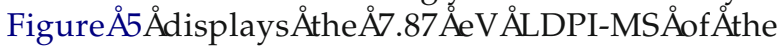
untagged Bis-GMA-methacryloyl overlayer along with the schematic of the bound species and the ion fragmentation scheme. By contrast, a parent ion peak at $m / z 640$ appeared in the $10.5 \mathrm{eV}$ LDPI-MS of this covalently ound Aompound 44$]$. The Åchematic Ån

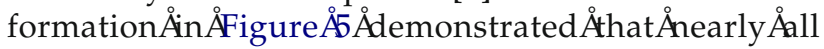
major ion peaks detected in this spectrum contained the methacrylate group. The highest mass peak from the Bis-GMA-methacryloyl overlayers occurred at $m / z 407$, cleaved at the bond of Si-C (bond f) and the bond a, not the mass peak at $\mathrm{m} / \mathrm{z} 640$ shown in the

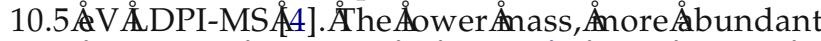

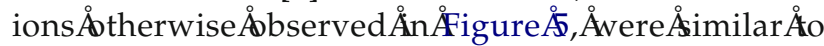
those observed in prior spectra.

The (Azo) $)_{2}$-Bis-GMA-methacryloyl overlayer was also examined by $7.87 \mathrm{eV}$ LDPI-MS (data not shown); the parent ion at $\mathrm{m} / \mathrm{z} 1057 \mathrm{did}$ not appear in the spectrum. No ion signal was observed above $\mathrm{m} / \mathrm{z} 250$, further confirming that the derivatized Azo tag did not contribute to the ionization of neutrals desorbed from the overlayer and consistent with the results for the

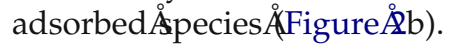

\section{Conclusions}

Analysis of Bis-GMA and several covalently tagged derivatives by laser desorption postionization mass spectrometry (LDPI-MS) provided further verification of the hypothesis that tagging of analytes with low IP 
compounds lowers the overall IP of the complex and allows its detection by $7.87 \mathrm{eV}$ single photon ionization

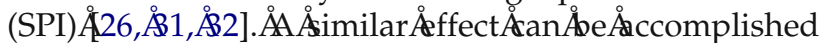

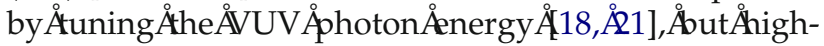
intensity, tunable VUV sources are currently only available at synchrotrons. Furthermore, chemical derivatization of specific classes of analytes within a complex mixture allows their selective detection via $7.87 \mathrm{eV}$ LDPI-MSÅ33].

Detection of both adsorbed and covalent bound species is feasible using this strategy, provided intact desorption of molecular analytes can be accomplished in a separate step. This points to another issue in

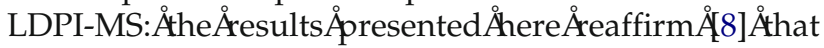
strong linear optical absorption of an analyte can lead to enhanced desorption yields by resonant laser de-

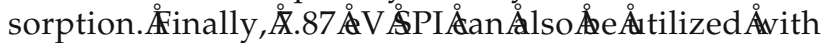
other desorption probes, including the accelerated atomic or cluster primary ions used in secondary ion mass \$pectrometry $\AA 12]$.

The simplified organic overlayer model of the dental composite $[4]$ ] after derivatization via $7.87 \mathrm{eV}$ LDPI-MS. This is a feasible method to further investigate the biodegradation mechanisms of the dental composite overlayer model system after aging in different simulated oral

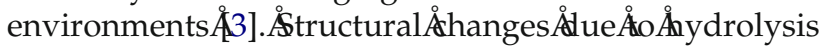
or other degradation process of the Benz-derivatized overlayers during exposure to oral environments should be revealed by $7.87 \mathrm{eV}$ LDPI-MS. This is a subject of ongoing work.

\section{Acknowledgments}

The authors acknowledge funding of this work by the National Institute of Dental and Craniofacial Research under grant number DE-07,979. The X-ray photoelectron spectroscopy measurements were made on equipment located in the Research Resources Center at UIC and funded by the National Science Foundation under grant CTS-0321202.

\section{References}

1. Ferracane, J. L. Current Trends in Dental Composites. Crit. Rev. Oral. Biol. Med. 1995, 6, 302-318.

2. Jones, F. H. Teeth and Bones: Applications of Surface Science to Dental Materials and Related Biomaterials. Surf. Sci. Rep. 2001, 42, 75-205.

3. Zhou, M.; Drummond, J. L.; Hanley, L. Barium and Strontium Leaching from Aged Glass Particle/Resin Matrix Dental Composites. Dent. Mater. 2005, 21, 145-155.

4. Zhou, M.; Wu, C.; Edirisinghe, P. D.; Drummond, J. L.; Hanley, L. Organic Overlayer Model of a Dental Composite Analyzed by Laser Desorption Postionization Mass Spectrometry and Photoemission. J. Biomed. Mater. Res. 2006, 77A, 1-10.

5. Wei, J.; Buriak, J. M.; Siuzdak, G. Desorption/Ionization Mass Spectrometry on Porous Silicon. Nature. 1999, 399, 243-246.

6. Pallix, J. B.; Schuehle, U.; Becker, C. H.; Huestis, D. L. Advantages of Single-Photon Ionization Over Multiphoton Ionization for Mass Spectrometric Surface Analysis of Bulk Organic Polymers. Anal. Chem. 1989, $61,805-811$.

7. Van Bramer, S. E.; Johnston, M. V. $10.5 \mathrm{eV}$ Photoionization Mass Spectrometry of Aliphatic Compounds. J. Am. Soc. Mass Spectrom. 1990, 1, 419-426.

8. Kinsel, G. R.; Lindner, J.; Grotemeyer, J.; Schlag, E. W. Absorption Effects in Laser Desorption of Neutral Organic Molecules. J. Phys. Chem. 1991, 95, 7824-7830

9. Schlag, E. W.; Grotemeyer, J.; Levine, R. D. Do Large Molecules Ionize? Chem. Phys. Lett. 1992, 190, 521-527.
10. Becker, C. H.; Wu, K. J. On the Photoionization of Large Molecules. J. Am. Soc. Mass Spectrom. 1995, 6, 883-888.

11. de Vries, M. S.; Hunziker, H. E. Vacuum UV Photoionization Mass Spectrometry of Small Polymers Using Jet Cooling. J. Photochem. Photobiol. A Chem. 1997, 106, 31-36.

12. Hanley, L.; Kornienko, O.; Ada, E. T.; Fuoco, E.; Trevor, J. L. Surface Mass Spectrometry of Molecular Species. J. Mass Spectrom. 1999, 34, 705-723.

13. Muhlberger, F.; Wieser, J.; Ulrich, A.; Zimmerman, R. Single Photon Ionization (SPI) Via Incoherent VUV-Excimer Light: Robust and Compact Time-of-Flight Mass Spectrometer for On-Line, Real-Time Process Gas Analysis. Anal. Chem. 2002, 74, 3790-3801.

14. Sykes, D. C.; Woods, E. I.; Smith, G. D.; Baer, T.; Miller, R. E. Thermal Vaporization-Vacuum Ultraviolet Laser Ionization Time-of-Flight Mass Spectrometry of Single Aerosol Particles. Anal. Chem. 2002, 74, $2048-$ 2052.

15. King, B. V.; Pellin, M. J.; Moore, J. F.; Veryovkin, I. V.; Tripa, C. E. Estimation of Useful Yield in Surface Analysis Using Single Photon Ionization. Appl. Surf. Sci. 2003, 203/204, 244-247.

16. Oktem, B.; Tolocka, M. P.; Johnston, M. V. On-Line Analysis of Organic Components in Fine and Ultrafine Particles by Photoionization Aerosol Mass Spectrometry. Anal. Chem. 2004, 76, 253-261.

17. Syage, J. A. Mechanism of $[\mathrm{M}+\mathrm{H}]^{+}$Formation in Photoionization Mass Spectrometry. J. Am. Soc. Mass Spectrom. 2004, 15, 1521-1533.

18. Jochims, H.-W.; Schwell, M.; Chotin, J.-L.; Clemino, M.; Dulieu, F.; Baumgärtel, H.; Leach, S. Photoion Mass Spectrometry of Five Amino Acids in the 6-22 eV Photon Energy Range. Chem. Phys. 2004, 298, 279-297.

19. Nash, D. G.; Liu, X. F.; Mysak, E. R.; Baer, T. Aerosol Particle Mass Spectrometry with Low Photon Energy Laser Ionization. Int. J. Mass Spectrom. Ion Processes. 2005, 241, 89-97.

20. Finch, J. W.; Toerne, K. A.; Schram, K. H.; Denton, M. B. Evaluation of a Hydrogen Laser Vacuum Ultraviolet Source for Photoionization Mass Spectrometry of Pharmaceuticals. Rapid Commun. Mass Spectrom. 2005, $19,15-22$.

21. Wilson, K. R.; Jimenez-Cruz, M.; Nicolas, C.; Belau, L.; Leone, S. R. Ahmed, M. Thermal Vaporization of Biological Nanoparticles: Fragment-Free Vacuum Ultraviolet Photoionization Mass Spectra of Tryptophan, Phenylalanine-Glycine-Glycine, and $\beta$-Carotene. J. Phys. Chem. A. 2006, 110, 2106-2113.

22. Boesl, U. Multiphoton Excitation and Mass-Selective Ion Detection for Neutral and Ion Spectroscopy. J. Phys. Chem. 1991, 95, 2949-2962.

23. Zenobi, R. In Situ Analysis of Surfaces and Mixtures by Laser Desorption Mass Spectrometry. Int. J. Mass Spectrom. Ion Processes. 1995, 145, 51-77.

24. Kalberer, M.; Morrical, B. D.; Sax, M.; Zenobi, R. Picogram Quantitation of Polycyclic Aromatic Hydrocarbons Adsorbed on Aerosol Particles by Two-Step Laser Mass Spectrometry. Anal. Chem. 2002, 74, 3492-3497.

25. King, B. V.; Savina, M. R.; Tripa, C. E.; Calaway, W. F.; Veryovkin, I. V. Moore, J. F.; Pellin, M. J. Single Photon Ionization of Self-Assembled Monolayers. Nucl. Instr. Methods Phys. Res. B. 2002, 190, 203-206.

26. Hanley, L.; Edirisinghe, P. D.; Calaway, W. F.; Veryovkin, I. V.; Pellin, M. J.; Moore, J. F. 7.87 eV Postionization of Peptides Containing Tryptophan or Derivatized with Fluorescein. Appl. Surf. Sci. 2006, 252, 6723-6726.

27. Anex, D. S.; de Vries, M. S.; Knebelkamp, A.; Bargon, J.; Wendt, H. R.; Hunziker, H. E. Resonance-Enhanced Two-Photon Ionization Time-ofFlight Spectroscopy of Cold Perfluorinated Polyethers and Their External and Internal van der Waals Dimers. Int. J. Mass Spectrom. Ion Processes. 1994, 131, 319-334.

28. Srinivasan, J. N.; Romano, L. J.; Levis, R. J. Velocity Distribution for a Laser-Vaporized, Anthracene-Labeled Nucleotide. J. Phys. Chem. 1995, 99, 13272.

29. Houston, C. T.; Reilly, J. P. Photoionization Studies of ChromophoreLabeled Amino Acids and Peptides. J. Phys. Chem. A. 2000, 104, 10383.

30. Fernandes-Whaley, M.; Muhlberger, F.; Whaley, A.; Adam, T.; Zimmermann, R.; Rohwer, E.; Walte, A. On-Line Derivatization for ResonanceEnhanced Multiphoton Ionization Time-of-Flight Mass Spectrometry: Detection of Aliphatic Aldehydes and Amines via Reactive Coupling of Aromatic Photo Ionization Labels. Anal. Chem. 2005, 77, 1-10.

31. Edirisinghe, P. D.; Lateef, S. S.; Crot, C. A.; Hanley, L.; Pellin, M. J. Calaway, W. F.; Moore, J. F. Derivatization of Surface Bound Peptides for Mass Spectrometric Detection via Threshold Single Photon Ionization. Anal. Chem. 2004, 76, 4267-4270.

32. Edirisinghe, P. D.; Moore, J. F.; Calaway, W. F.; Veryovkin, I. V.; Pellin, M. J.; Hanley, L. Vacuum Ultraviolet Postionization of Aromatic Groups Covalently Bound to Peptides. Anal. Chem. 2006, 78, 5876-5883.

33. Edirisinghe, P. D.; Moore, J. F.; Skinner-Nemec, K. A.; Lindberg, C. Giometti, C. S.; Veryovkin, I. V.; Hunt, J. E.; Pellin, M. J.; Hanley, L. Detection of in-Situ Derivatized Peptides in Microbial Biofilms by Laser Desorption 7.87 eV Postionizaton Mass Spectrometry. Anal. Chem. 2007, 79, 508-514.

34. Wu C. Laser Desorption Postionization Time-of-Flight Instrumentation and Application to Dental Materials. M.Sc. Thesis, University of Illinois at Chicago; 2005.

35. Lias, S. G.; Bartmess, J. E.; Liebman, J. F.; Holmes, J. L.; Levin, R. D.; Mallard, W. G. Gas-Phase Ion and Neutral Thermochemistry. J. Phys. Chem. Ref. Data. 1988, 17, 18-25. 
36. webbook.nist.gov, accessed 11/06.

37. Kan, R. O. Organic Photochemistry; McGraw Hill: New York, NY, 1966

38. Hanley, L.; Guo, X.; Yates, J. T., Jr. Photolysis of Azomethane adsorbed on Pd(111). Surf. Sci. 1990, 232, 129-137.

39. Shen, Z.; Thomas, J. J.; Averbuj, C.; Brook, M.; Engelhard, M.; Crowell, J. E.; Finn, M. G.; Siuzdak, G. Porous Silicon as a Versatile Platform for Desorption/Ionization Mass Spectrometry. Anal. Chem. 2001, 73, 612-619.

40. Choi, Y.; Zachary, A.; Tepavcevic, S.; Wu, C.; Hanley, L. Polyatomicity and Kinetic Energy Effects on Surface Polymerization by Ion-
Assisted Deposition. Int. J. Mass Spectrom. Ion Processes. 2005, 241, 139-147.

41. Luo, G.; Chen, Y.; Daniels, H.; Dubrow, R.; Vertes, A. Internal Energy Transfer in Laser Desorption/Ionization from Silicon Nanowires. J. Phys. Chem. B. 2006, 110, 13381-13386.

42. Kornienko, O.; Ada, E. T.; Tinka, J.; Wijesundara, M. B. J.; Hanley, L. Organic Surface Analysis by Two Laser Ion Trap Mass Spectrometry. Part II. Improved Desorption/Photoionization Configuration. Anal. Chem. 1998, 70, 1208-1213. 